\title{
Unexpected Influence of the Counteranion in the $\square^{2}$ vs. $\square^{3}$ Hapticity of Polydentate $N$-donor Ligands in $\left[\operatorname{Rh}^{\mathbf{I}}(N \text {-ligand }) L_{2}\right]^{+}$Complexes.
}

\author{
Gabriel Aullón, Glòria Esquius, Agustí Lledós, * Feliu Maseras, \\ Josefina Pons, and Josep Ros*
}

\section{Supporting Information}

25 pages

\section{Table of Contents}

Tables S. Atomic coordinates of the optimized structures:
S1: $\left[\mathrm{Rh}\left(\square^{3}-\mathrm{N}_{3}{ }^{H}\right)(\mathrm{cod})\right]^{+}: \mathbf{1 a}(\mathrm{pp} .2)$
S10: $\left[\mathrm{Rh}\left(\square^{3}-\mathrm{N}_{3}{ }^{M e}\right)(\mathrm{CO})\right]^{+}:(\mathrm{pp} .11)$
S2: $\left[\mathrm{Rh}\left(\square^{2}-\mathrm{N}_{3}{ }^{H}\right)(\operatorname{cod})\right]^{+}: \mathbf{1 b}(\mathrm{pp} .3)$
S11: $\left[\mathrm{Rh}\left(\mathrm{N}_{3}{ }^{M e}\right)(\operatorname{cod})\right]^{+}:$ts $(\mathrm{pp} .12)$
S3: $\left[\mathrm{Rh}\left(\square^{3}-\mathrm{N}_{3}{ }^{H}\right)(\mathrm{CO})_{2}\right]^{+}$: $\mathbf{1 a}(\mathrm{pp} .4)$
S12: $\left[\mathrm{Rh}\left(\mathrm{N}_{3}{ }^{M e}\right)(\mathrm{CO})_{2}\right]^{+}:$ts (pp.13)
S4: $\left[\mathrm{Rh}\left(\square^{2}-\mathrm{N}_{3}{ }^{H}\right)(\mathrm{CO})_{2}\right]^{+}: \mathbf{1 b}(\mathrm{pp} .5)$
S13: $\left[\mathrm{Rh}\left(\square^{3}-\mathrm{Tp}^{H}\right)(\mathrm{CO})_{2}\right]^{+}$: SPy (pp.14)
S5: $\left[\mathrm{Rh}\left(\square^{3}-\mathrm{N}_{3}^{M e}\right)(\mathrm{cod})\right]^{+}: \mathbf{1 a}(\mathrm{pp} .6)$
S14: $\left[\mathrm{Rh}\left(\square^{2}-\mathrm{Tp}^{H}\right)(\mathrm{CO})_{2}\right]^{+}: \mathrm{SP} 1$ (pp.15)
S6: $\left[\mathrm{Rh}\left(\square^{2}-\mathrm{N}_{3}^{M e}\right)(\operatorname{cod})\right]^{+}: \mathbf{1 b}(\mathrm{pp} .7)$
S15: $\left[\mathrm{Rh}\left(\square^{2}-\mathrm{Tp}^{H}\right)(\mathrm{CO})_{2}\right]^{+}: \mathrm{SP} 2$ (pp.16)
S7: $\left[\mathrm{Rh}\left(\square^{3}-\mathrm{N}_{3}{ }^{M e}\right)(\mathrm{CO})_{2}\right]^{+}: \mathbf{1 a}(\mathrm{pp} .8)$
S16: $\left[\mathrm{Rh}\left(\square^{3}-\mathrm{Tp}^{M e}\right)(\mathrm{CO})_{2}\right]^{+}$: SPy (pp.17)
S8: $\left[\mathrm{Rh}\left(\square^{2}-\mathrm{N}_{3}{ }^{M e}\right)(\mathrm{CO})_{2}\right]^{+}: \mathbf{1 b}$ (pp.9)
S17: $\left[\mathrm{Rh}\left(\square^{2}-\mathrm{Tp}{ }^{M e}\right)(\mathrm{CO})_{2}\right]^{+}: \mathrm{SP} 2$ (pp.18)
S9: $\left[\mathrm{Rh}\left(\square^{3}-\mathrm{N}_{3}{ }^{H}\right)(\mathrm{CO})\right]^{+}(\mathrm{pp} .10)$

Tables C. Main data for $\mathrm{d}^{8}-\mathrm{MTpL}_{2}$ complexes found in Cambridge Structural Database

C1: Summary and scheme (pp.19)

C2: SPy: $\square^{3}($ pp.20)

C3: TBP: $\square^{3}$ (pp.21)
C4: SP1: $\square^{2}($ pp.22-24)

C5: SP2: $\square^{2}(\mathrm{pp} .25)$ 
Table S1. Optimized structure of $\left[\operatorname{Rh}\left(\square^{3}-\mathrm{N}_{3}{ }^{H}\right)(\operatorname{cod})\right]^{+}$

\begin{tabular}{|c|c|c|c|}
\hline $\mathrm{Rh}$ & 1.000000 & 0.000000 & 1.000000 \\
\hline $\mathrm{X}$ & 0.998441 & 1.410059 & -0.485576 \\
\hline $\mathrm{X}$ & 0.998441 & -1.410059 & -0.485576 \\
\hline C & 0.296972 & 1.409692 & -0.485189 \\
\hline $\mathrm{C}$ & 1.699911 & 1.410427 & -0.485963 \\
\hline $\mathrm{C}$ & 0.296972 & -1.409692 & -0.485189 \\
\hline C & 1.699911 & -1.410427 & -0.485963 \\
\hline $\mathrm{H}$ & -0.189081 & 2.200743 & 0.084910 \\
\hline $\mathrm{H}$ & 2.185764 & 2.201987 & 0.083599 \\
\hline $\mathrm{H}$ & -0.189081 & -2.200743 & 0.084910 \\
\hline $\mathrm{H}$ & 2.185764 & -2.201987 & 0.083599 \\
\hline C & -0.558360 & 0.779454 & -1.576733 \\
\hline $\mathrm{C}$ & 2.554697 & 0.781085 & -1.578451 \\
\hline $\mathrm{C}$ & -0.558360 & -0.779454 & -1.576733 \\
\hline $\mathrm{C}$ & 2.554697 & -0.781085 & -1.578451 \\
\hline $\mathrm{H}$ & -0.220234 & 1.153122 & -2.551275 \\
\hline $\mathrm{H}$ & -1.588487 & 1.137742 & -1.457759 \\
\hline $\mathrm{H}$ & 2.215105 & 1.154398 & -2.552619 \\
\hline $\mathrm{H}$ & 3.584579 & 1.140452 & -1.460614 \\
\hline $\mathrm{H}$ & -0.220234 & -1.153122 & -2.551275 \\
\hline $\mathrm{H}$ & -1.588487 & -1.137742 & -1.457759 \\
\hline $\mathrm{H}$ & 2.215105 & -1.154398 & -2.552619 \\
\hline $\mathrm{H}$ & 3.584579 & -1.140452 & -1.460614 \\
\hline $\mathrm{N}$ & 0.739840 & 1.459737 & 2.587527 \\
\hline $\mathrm{N}$ & 0.739840 & -1.459737 & 2.587527 \\
\hline $\mathrm{N}$ & 1.677407 & 1.809143 & 3.508421 \\
\hline C & 1.187154 & 2.758538 & 4.367246 \\
\hline $\mathrm{C}$ & -0.115144 & 3.028681 & 3.982559 \\
\hline C & -0.349152 & 2.195524 & 2.869539 \\
\hline $\mathrm{H}$ & 1.786784 & 3.160477 & 5.167518 \\
\hline $\mathrm{H}$ & -0.799043 & 3.725995 & 4.438170 \\
\hline $\mathrm{H}$ & -1.241799 & 2.099763 & 2.273252 \\
\hline $\mathrm{N}$ & 1.677407 & -1.809143 & 3.508421 \\
\hline C & 1.187154 & -2.758538 & 4.367246 \\
\hline C & -0.115144 & -3.028681 & 3.982559 \\
\hline C & -0.349152 & -2.195524 & 2.869539 \\
\hline $\mathrm{H}$ & 1.786784 & -3.160477 & 5.167518 \\
\hline $\mathrm{H}$ & -0.799043 & -3.725995 & 4.438170 \\
\hline $\mathrm{H}$ & -1.241799 & -2.099763 & 2.273252 \\
\hline $\mathrm{C}$ & 3.052278 & 1.233419 & 3.570574 \\
\hline $\mathrm{C}$ & 3.052278 & -1.233419 & 3.570574 \\
\hline $\mathrm{H}$ & 3.739762 & 1.981004 & 3.159585 \\
\hline $\mathrm{H}$ & 3.739762 & -1.981004 & 3.159585 \\
\hline $\mathrm{H}$ & 3.273665 & 1.118498 & 4.637443 \\
\hline $\mathrm{H}$ & 3.273665 & -1.118498 & 4.637443 \\
\hline $\mathrm{N}$ & 3.211015 & 0.000000 & 2.830320 \\
\hline $\mathrm{C}$ & 4.353843 & 0.000000 & 1.866427 \\
\hline C & 5.746079 & 0.000000 & 2.513387 \\
\hline $\mathrm{H}$ & 4.226166 & 0.876060 & 1.223539 \\
\hline $\mathrm{H}$ & 4.226166 & -0.876060 & 1.223539 \\
\hline $\mathrm{H}$ & 6.536985 & 0.000000 & 1.754874 \\
\hline $\mathrm{H}$ & 5.893569 & -0.887198 & 3.139486 \\
\hline $\mathrm{H}$ & 5.893569 & 0.887198 & 3.139486 \\
\hline
\end{tabular}


Table S2. Optimized structure of $\left[\mathrm{Rh}\left(\square^{2}-\mathrm{N}_{3}{ }^{H}\right)(\operatorname{cod})\right]^{+}$

\begin{tabular}{|c|c|c|c|}
\hline $\mathrm{Rh}$ & 1.000000 & 0.000000 & 1.000000 \\
\hline $\mathrm{X}$ & 0.999851 & 1.423878 & -0.499673 \\
\hline $\mathrm{X}$ & 0.999851 & -1.423878 & -0.499673 \\
\hline $\mathrm{C}$ & 0.301168 & 1.423843 & -0.499636 \\
\hline $\mathrm{C}$ & 1.698533 & 1.423912 & -0.499710 \\
\hline $\mathrm{C}$ & 0.301168 & -1.423843 & -0.499636 \\
\hline $\mathrm{C}$ & 1.698533 & -1.423912 & -0.499710 \\
\hline $\mathrm{H}$ & -0.187311 & 2.207281 & 0.078700 \\
\hline $\mathrm{H}$ & 2.186995 & 2.207400 & 0.078576 \\
\hline $\mathrm{H}$ & -0.187311 & -2.207281 & 0.078700 \\
\hline $\mathrm{H}$ & 2.186995 & -2.207400 & 0.078576 \\
\hline $\mathrm{C}$ & -0.556815 & 0.781383 & -1.580212 \\
\hline $\mathrm{C}$ & 2.556467 & 0.781538 & -1.580375 \\
\hline $\mathrm{C}$ & -0.556815 & -0.781383 & -1.580212 \\
\hline $\mathrm{C}$ & 2.556467 & -0.781538 & -1.580375 \\
\hline $\mathrm{H}$ & -0.222231 & 1.150263 & -2.557561 \\
\hline $\mathrm{H}$ & -1.587074 & 1.137759 & -1.458585 \\
\hline $\mathrm{H}$ & 2.221743 & 1.150385 & -2.557689 \\
\hline $\mathrm{H}$ & 3.586703 & 1.138017 & -1.458856 \\
\hline $\mathrm{H}$ & -0.222231 & -1.150263 & -2.557561 \\
\hline $\mathrm{H}$ & -1.587074 & -1.137759 & -1.458585 \\
\hline $\mathrm{H}$ & 2.221743 & -1.150385 & -2.557689 \\
\hline $\mathrm{H}$ & 3.586703 & -1.138017 & -1.458856 \\
\hline $\mathrm{N}$ & 0.949534 & 1.491021 & 2.550200 \\
\hline $\mathrm{N}$ & 0.949534 & -1.491021 & 2.550200 \\
\hline $\mathrm{N}$ & 2.003352 & 1.861383 & 3.331196 \\
\hline $\mathrm{C}$ & 1.623691 & 2.837584 & 4.217444 \\
\hline $\mathrm{C}$ & 0.282496 & 3.100945 & 3.996585 \\
\hline $\mathrm{C}$ & -0.095077 & 2.236885 & 2.947180 \\
\hline $\mathrm{H}$ & 2.321759 & 3.260294 & 4.921435 \\
\hline $\mathrm{H}$ & -0.338286 & 3.813027 & 4.515384 \\
\hline $\mathrm{H}$ & -1.058043 & 2.128392 & 2.475469 \\
\hline $\mathrm{N}$ & 2.003352 & -1.861383 & 3.331196 \\
\hline $\mathrm{C}$ & 1.623691 & -2.837584 & 4.217444 \\
\hline $\mathrm{C}$ & 0.282496 & -3.100945 & 3.996585 \\
\hline $\mathrm{C}$ & -0.095077 & -2.236885 & 2.947180 \\
\hline $\mathrm{H}$ & 2.321759 & -3.260294 & 4.921435 \\
\hline $\mathrm{H}$ & -0.338286 & -3.813027 & 4.515384 \\
\hline $\mathrm{H}$ & -1.058043 & -2.128392 & 2.475469 \\
\hline $\mathrm{C}$ & 3.356017 & 1.246705 & 3.186639 \\
\hline $\mathrm{C}$ & 3.356017 & -1.246705 & 3.186639 \\
\hline $\mathrm{H}$ & 4.047488 & 2.001826 & 3.570292 \\
\hline $\mathrm{H}$ & 4.047488 & -2.001826 & 3.570292 \\
\hline $\mathrm{H}$ & 3.526590 & 1.123668 & 2.113655 \\
\hline $\mathrm{H}$ & 3.526590 & -1.123668 & 2.113655 \\
\hline $\mathrm{N}$ & 3.579923 & 0.000000 & 3.857213 \\
\hline $\mathrm{C}$ & 3.718633 & 0.000000 & 5.331469 \\
\hline $\mathrm{C}$ & 5.184499 & 0.000000 & 5.785241 \\
\hline $\mathrm{H}$ & 3.190558 & -0.871848 & 5.735254 \\
\hline $\mathrm{H}$ & 3.190558 & 0.871848 & 5.735254 \\
\hline $\mathrm{H}$ & 5.256308 & 0.000000 & 6.878394 \\
\hline $\mathrm{H}$ & 5.710502 & 0.885172 & 5.411166 \\
\hline $\mathrm{H}$ & 5.710502 & -0.885172 & 5.411166 \\
\hline
\end{tabular}


Table S3. Optimized structure of $\left[\mathrm{Rh}\left(\square^{3}-\mathrm{N}_{3}{ }^{H}\right)(\mathrm{CO})_{2}\right]^{+}$

\begin{tabular}{|c|c|c|c|}
\hline $\mathrm{Rh}$ & 1.000000 & 0.000000 & 1.000000 \\
\hline $\mathrm{C}$ & 0.861658 & 1.348031 & -0.312609 \\
\hline C & 0.861658 & -1.348031 & -0.312609 \\
\hline $\mathrm{O}$ & 0.769648 & 2.190551 & -1.082060 \\
\hline $\mathrm{O}$ & 0.769648 & -2.190551 & -1.082060 \\
\hline $\mathrm{N}$ & 0.942590 & 1.452754 & 2.565644 \\
\hline $\mathrm{N}$ & 0.942590 & -1.452754 & 2.565644 \\
\hline $\mathrm{N}$ & 1.989271 & 1.811271 & 3.356228 \\
\hline $\mathrm{C}$ & 1.603268 & 2.768434 & 4.255106 \\
\hline C & 0.260875 & 3.032431 & 4.032108 \\
\hline $\mathrm{C}$ & -0.109481 & 2.188133 & 2.968375 \\
\hline $\mathrm{H}$ & 2.294752 & 3.182236 & 4.970766 \\
\hline $\mathrm{H}$ & -0.362525 & 3.734296 & 4.56155 \\
\hline $\mathrm{H}$ & -1.067581 & 2.081262 & 2.48653 \\
\hline $\mathrm{N}$ & 1.989271 & -1.811271 & 3.356228 \\
\hline C & 1.603268 & -2.768434 & 4.255106 \\
\hline $\mathrm{C}$ & 0.260875 & -3.032431 & 4.032108 \\
\hline $\mathrm{C}$ & -0.109481 & -2.188133 & 2.96837 \\
\hline $\mathrm{H}$ & 2.294752 & -3.182236 & 4.97076 \\
\hline $\mathrm{H}$ & -0.362525 & -3.734296 & 4.56155 \\
\hline $\mathrm{H}$ & -1.067581 & -2.081262 & 2.48653 \\
\hline $\mathrm{C}$ & 3.363375 & 1.232742 & 3.24759 \\
\hline C & 3.363375 & -1.232742 & 3.24759 \\
\hline $\mathrm{H}$ & 3.992647 & 1.981190 & 2.754404 \\
\hline $\mathrm{H}$ & 3.992647 & -1.981190 & 2.75440 \\
\hline $\mathrm{H}$ & 3.713564 & 1.115881 & 4.27867 \\
\hline $\mathrm{H}$ & 3.713564 & -1.115881 & 4.27867 \\
\hline $\mathrm{N}$ & 3.420934 & 0.000000 & 2.49179 \\
\hline C & 4.420495 & 0.000000 & 1.374164 \\
\hline $\mathrm{C}$ & 5.887442 & 0.000000 & 1.823635 \\
\hline $\mathrm{H}$ & 4.204771 & 0.876670 & 0.75580 \\
\hline $\mathrm{H}$ & 4.204771 & -0.876670 & 0.75580 \\
\hline $\mathrm{H}$ & 6.566462 & 0.000000 & 0.96374 \\
\hline $\mathrm{H}$ & 6.119393 & -0.887493 & 2.422840 \\
\hline $\mathrm{H}$ & 6.119393 & 0.887493 & 2.42284 \\
\hline
\end{tabular}


Table S4. Optimized structure of $\left[\mathrm{Rh}\left(\square^{2}-\mathrm{N}_{3}{ }^{H}\right)(\mathrm{CO})_{2}\right]^{+}$

$\begin{array}{rrrr}\mathrm{Rh} & -0.825352 & 0.000000 & -1.249774 \\ \mathrm{C} & -1.034490 & 1.363384 & -2.552945 \\ \mathrm{C} & -1.034490 & -1.363384 & -2.552945 \\ \mathrm{O} & -1.156272 & 2.204027 & -3.317064 \\ \mathrm{O} & -1.156272 & -2.204027 & -3.317064 \\ \mathrm{~N} & -0.630614 & 1.477704 & 0.267620 \\ \mathrm{~N} & -0.630614 & -1.477704 & 0.267620 \\ \mathrm{~N} & 0.535294 & 1.854439 & 0.864930 \\ \mathrm{C} & 0.292725 & 2.829146 & 1.795867 \\ \mathrm{C} & -1.069974 & 3.083766 & 1.794079 \\ \mathrm{C} & -1.607499 & 2.216488 & 0.823712 \\ \mathrm{H} & 1.090704 & 3.260135 & 2.378172 \\ \mathrm{H} & -1.603059 & 3.793802 & 2.405039 \\ \mathrm{H} & -2.632619 & 2.098759 & 0.512473 \\ \mathrm{~N} & 0.535294 & -1.854439 & 0.864930 \\ \mathrm{C} & 0.292725 & -2.829146 & 1.795867 \\ \mathrm{C} & -1.069974 & -3.083766 & 1.794079 \\ \mathrm{C} & -1.607499 & -2.216488 & 0.823712 \\ \mathrm{H} & 1.090704 & -3.260135 & 2.378172 \\ \mathrm{H} & -1.603059 & -3.793802 & 2.405039 \\ \mathrm{H} & -2.632619 & -2.098759 & 0.512473 \\ \mathrm{C} & 1.855022 & 1.246046 & 0.504130 \\ \mathrm{C} & 1.855022 & -1.246046 & 0.504130 \\ \mathrm{H} & 2.593322 & 2.004130 & 0.779342 \\ \mathrm{H} & 2.593322 & -2.004130 & 0.779342 \\ \mathrm{H} & 1.857575 & 1.133979 & -0.583507 \\ \mathrm{H} & 1.857575 & -1.133979 & -0.583507 \\ \mathrm{~N} & 2.180410 & 0.000000 & 1.127911 \\ \mathrm{C} & 2.581936 & 0.000000 & 2.554765 \\ \mathrm{C} & 4.104919 & 0.000000 & 2.738081 \\ \mathrm{H} & 2.133772 & -0.871917 & 3.045531 \\ \mathrm{H} & 2.133772 & 0.871917 & 3.045531 \\ \mathrm{H} & 4.371951 & 0.000000 & 3.800434 \\ \mathrm{H} & 4.555435 & 0.885137 & 2.276057 \\ \mathrm{H} & 4.555435 & -0.885137 & 2.276057 \\ & & & \end{array}$


Table S5. Optimized structure of $\left[\mathrm{Rh}\left(\square^{3}-\mathrm{N}_{3}{ }^{M e}\right)(\operatorname{cod})\right]^{+}$

\begin{tabular}{|c|c|c|c|}
\hline $\mathrm{Rh}$ & 1.000000 & 0.000000 & 1.000000 \\
\hline $\mathrm{X}$ & 0.998827 & 1.406778 & -0.488124 \\
\hline $\mathrm{X}$ & 0.998827 & -1.406778 & -0.488124 \\
\hline $\mathrm{C}$ & 0.296907 & 1.406502 & -0.487832 \\
\hline $\mathrm{C}$ & 1.700746 & 1.407054 & -0.488416 \\
\hline $\mathrm{C}$ & 0.296907 & -1.406502 & -0.487832 \\
\hline $\mathrm{C}$ & 1.700746 & -1.407054 & -0.488416 \\
\hline $\mathrm{H}$ & -0.185664 & 2.205438 & 0.072919 \\
\hline $\mathrm{H}$ & 2.183156 & 2.206371 & 0.071933 \\
\hline $\mathrm{H}$ & -0.185664 & -2.205438 & 0.072919 \\
\hline $\mathrm{H}$ & 2.183156 & -2.206371 & 0.071933 \\
\hline $\mathrm{C}$ & -0.555773 & 0.779133 & -1.583739 \\
\hline $\mathrm{C}$ & 2.553008 & 0.780357 & -1.585034 \\
\hline $\mathrm{C}$ & -0.555773 & -0.779133 & -1.583739 \\
\hline $\mathrm{C}$ & 2.553008 & -0.780357 & -1.585034 \\
\hline $\mathrm{H}$ & -0.214510 & 1.153892 & -2.557008 \\
\hline $\mathrm{H}$ & -1.586162 & 1.138550 & -1.468521 \\
\hline $\mathrm{H}$ & 2.210640 & 1.154846 & -2.558018 \\
\hline $\mathrm{H}$ & 3.583208 & 1.140584 & -1.470673 \\
\hline $\mathrm{H}$ & -0.214510 & -1.153892 & -2.557008 \\
\hline $\mathrm{H}$ & -1.586162 & -1.138550 & -1.468521 \\
\hline $\mathrm{H}$ & 2.210640 & -1.154846 & -2.558018 \\
\hline $\mathrm{H}$ & 3.583208 & -1.140584 & -1.470673 \\
\hline $\mathrm{N}$ & 0.729158 & 1.442105 & 2.622030 \\
\hline $\mathrm{N}$ & 0.729158 & -1.442105 & 2.622030 \\
\hline $\mathrm{N}$ & 1.752453 & 1.788717 & 3.464980 \\
\hline $\mathrm{C}$ & 1.335305 & 2.711454 & 4.401330 \\
\hline $\mathrm{C}$ & -0.002072 & 2.952593 & 4.134666 \\
\hline $\mathrm{C}$ & -0.345737 & 2.147430 & 3.023028 \\
\hline $\mathrm{C}$ & 2.234805 & 3.275453 & 5.454250 \\
\hline $\mathrm{H}$ & -0.651997 & 3.624109 & 4.671906 \\
\hline $\mathrm{C}$ & -1.682557 & 2.053863 & 2.353961 \\
\hline $\mathrm{N}$ & 1.752453 & -1.788717 & 3.464980 \\
\hline $\mathrm{C}$ & 1.335305 & -2.711454 & 4.401330 \\
\hline $\mathrm{C}$ & -0.002072 & -2.952593 & 4.134666 \\
\hline $\mathrm{C}$ & -0.345737 & -2.147430 & 3.023028 \\
\hline $\mathrm{C}$ & 2.234805 & -3.275453 & 5.454250 \\
\hline $\mathrm{H}$ & -0.651997 & -3.624109 & 4.671906 \\
\hline $\mathrm{C}$ & -1.682557 & -2.053863 & 2.353961 \\
\hline $\mathrm{C}$ & 3.123863 & 1.235947 & 3.370935 \\
\hline $\mathrm{C}$ & 3.123863 & -1.235947 & 3.370935 \\
\hline $\mathrm{H}$ & 3.758746 & 1.987478 & 2.887480 \\
\hline $\mathrm{H}$ & 3.758746 & -1.987478 & 2.887480 \\
\hline $\mathrm{H}$ & 3.475360 & 1.115593 & 4.402229 \\
\hline $\mathrm{H}$ & 3.475360 & -1.115593 & 4.402229 \\
\hline $\mathrm{N}$ & 3.222131 & 0.000000 & 2.605515 \\
\hline $\mathrm{C}$ & 4.379869 & 0.000000 & 1.655889 \\
\hline $\mathrm{C}$ & 5.767718 & 0.000000 & 2.314296 \\
\hline $\mathrm{H}$ & 4.259858 & 0.876534 & 1.013253 \\
\hline $\mathrm{H}$ & 4.259858 & -0.876534 & 1.013253 \\
\hline $\mathrm{H}$ & 6.565055 & 0.000000 & 1.562577 \\
\hline $\mathrm{H}$ & 5.911161 & -0.887468 & 2.940915 \\
\hline $\mathrm{H}$ & 5.911161 & 0.887468 & 2.940915 \\
\hline $\mathrm{H}$ & 3.101268 & 3.779159 & 5.011734 \\
\hline $\mathrm{H}$ & 1.710445 & 4.008627 & 6.076986 \\
\hline $\mathrm{H}$ & 2.613891 & 2.490770 & 6.118225 \\
\hline $\mathrm{H}$ & 3.101268 & -3.779159 & 5.011734 \\
\hline $\mathrm{H}$ & 1.710445 & -4.008627 & 6.076986 \\
\hline $\mathrm{H}$ & 2.613891 & -2.490770 & 6.118225 \\
\hline $\mathrm{H}$ & -1.885792 & 2.948000 & 1.756110 \\
\hline $\mathrm{H}$ & -1.728282 & 1.186530 & 1.687737 \\
\hline $\mathrm{H}$ & -2.484756 & 1.953485 & 3.091941 \\
\hline $\mathrm{H}$ & -1.885792 & -2.948000 & 1.756110 \\
\hline $\mathrm{H}$ & -1.728282 & -1.186530 & 1.687737 \\
\hline $\mathrm{H}$ & -2.484756 & -1.953485 & 3.091941 \\
\hline
\end{tabular}


Table S6. Optimized structure of $\left[\mathrm{Rh}\left(\square^{2}-\mathrm{N}_{3}{ }^{M e}\right)(\mathrm{cod})\right]^{+}$

\begin{tabular}{|c|c|c|c|}
\hline $\mathrm{Rh}$ & -0.543919 & 0.000000 & -0.985213 \\
\hline $\mathrm{X}$ & -0.543219 & 1.422639 & -2.488029 \\
\hline $\mathrm{X}$ & -0.543219 & -1.422639 & -2.488029 \\
\hline $\mathrm{C}$ & -1.241740 & 1.422802 & -2.488201 \\
\hline $\mathrm{C}$ & 0.155301 & 1.422477 & -2.487858 \\
\hline $\mathrm{C}$ & -1.241740 & -1.422802 & -2.488201 \\
\hline C & 0.155301 & -1.422477 & -2.487858 \\
\hline $\mathrm{H}$ & -1.727843 & 2.210280 & -1.914598 \\
\hline $\mathrm{H}$ & 0.641488 & 2.209730 & -1.914017 \\
\hline $\mathrm{H}$ & -1.727843 & -2.210280 & -1.914598 \\
\hline $\mathrm{H}$ & 0.641488 & -2.209730 & -1.914017 \\
\hline $\mathrm{C}$ & -2.099859 & 0.781526 & -3.569539 \\
\hline $\mathrm{C}$ & 1.013652 & 0.780803 & -3.568775 \\
\hline $\mathrm{C}$ & -2.099859 & -0.781526 & -3.569539 \\
\hline $\mathrm{C}$ & 1.013652 & -0.780803 & -3.568775 \\
\hline $\mathrm{H}$ & -1.765533 & 1.150928 & -4.546983 \\
\hline $\mathrm{H}$ & -3.130106 & 1.138457 & -3.447807 \\
\hline $\mathrm{H}$ & 0.679977 & 1.150360 & -4.546383 \\
\hline $\mathrm{H}$ & 2.044006 & 1.137255 & -3.446537 \\
\hline $\mathrm{H}$ & -1.765533 & -1.150928 & -4.546983 \\
\hline $\mathrm{H}$ & -3.130106 & -1.138457 & -3.447807 \\
\hline $\mathrm{H}$ & 0.679977 & -1.150360 & -4.546383 \\
\hline $\mathrm{H}$ & 2.044006 & -1.137255 & -3.446537 \\
\hline $\mathrm{N}$ & -0.542714 & 1.484368 & 0.594625 \\
\hline $\mathrm{N}$ & -0.542714 & -1.484368 & 0.594625 \\
\hline $\mathrm{N}$ & 0.592142 & 1.849711 & 1.277547 \\
\hline C & 0.304949 & 2.809443 & 2.224363 \\
\hline $\mathrm{C}$ & -1.055670 & 3.053190 & 2.131059 \\
\hline $\mathrm{C}$ & -1.551821 & 2.211491 & 1.106797 \\
\hline $\mathrm{C}$ & 1.330392 & 3.411150 & 3.130441 \\
\hline $\mathrm{H}$ & -1.624177 & 3.750066 & 2.725251 \\
\hline $\mathrm{C}$ & -2.965191 & 2.101163 & 0.623047 \\
\hline $\mathrm{N}$ & 0.592142 & -1.849711 & 1.277547 \\
\hline $\mathrm{C}$ & 0.304949 & -2.809443 & 2.224363 \\
\hline C & -1.055670 & -3.053190 & 2.131059 \\
\hline $\mathrm{C}$ & -1.551821 & -2.211491 & 1.106797 \\
\hline C & 1.330392 & -3.411150 & 3.130441 \\
\hline $\mathrm{H}$ & -1.624177 & -3.750066 & 2.725251 \\
\hline $\mathrm{C}$ & -2.965191 & -2.101163 & 0.623047 \\
\hline $\mathrm{C}$ & 1.899012 & 1.236174 & 0.963424 \\
\hline C & 1.899012 & -1.236174 & 0.963424 \\
\hline $\mathrm{H}$ & 2.657982 & 1.983937 & 1.211107 \\
\hline $\mathrm{H}$ & 2.657982 & -1.983937 & 1.211107 \\
\hline $\mathrm{H}$ & 1.901053 & 1.087180 & -0.120241 \\
\hline $\mathrm{H}$ & 1.901053 & -1.087180 & -0.120241 \\
\hline $\mathrm{N}$ & 2.179736 & 0.000000 & 1.666832 \\
\hline $\mathrm{C}$ & 3.345051 & 0.000000 & 2.583697 \\
\hline C & 4.718399 & 0.000000 & 1.892027 \\
\hline $\mathrm{H}$ & 3.251838 & -0.874610 & 3.236918 \\
\hline $\mathrm{H}$ & 3.251838 & 0.874610 & 3.236918 \\
\hline $\mathrm{H}$ & 5.531981 & 0.000000 & 2.626507 \\
\hline $\mathrm{H}$ & 4.843647 & 0.886541 & 1.259782 \\
\hline $\mathrm{H}$ & 4.843647 & -0.886541 & 1.259782 \\
\hline $\mathrm{H}$ & 2.161912 & 3.839947 & 2.561153 \\
\hline $\mathrm{H}$ & 0.900571 & 4.211716 & 3.741775 \\
\hline $\mathrm{H}$ & 1.747647 & 2.662331 & 3.812020 \\
\hline $\mathrm{H}$ & 2.161912 & -3.839947 & 2.561153 \\
\hline $\mathrm{H}$ & 0.900571 & -4.211716 & 3.741775 \\
\hline $\mathrm{H}$ & 1.747647 & -2.662331 & 3.812020 \\
\hline $\mathrm{H}$ & -3.280656 & 3.019076 & 0.116403 \\
\hline $\mathrm{H}$ & -3.075586 & 1.272946 & -0.084512 \\
\hline $\mathrm{H}$ & -3.653661 & 1.924482 & 1.455852 \\
\hline $\mathrm{H}$ & -3.280656 & -3.019076 & 0.116403 \\
\hline $\mathrm{H}$ & -3.075586 & -1.272946 & -0.084512 \\
\hline $\mathrm{H}$ & -3.653661 & -1.924482 & 1.455852 \\
\hline
\end{tabular}


Table S7. Optimized structure of $\left[\mathrm{Rh}\left(\square^{3}-\mathrm{N}_{3} M e\right)(\mathrm{CO})_{2}\right]^{+}$

\begin{tabular}{|c|c|c|c|}
\hline $\mathrm{Rh}$ & -0.445065 & 0.000000 & -1.260806 \\
\hline $\mathrm{C}$ & -0.514487 & 1.347607 & -2.575376 \\
\hline $\mathrm{C}$ & -0.514487 & -1.347607 & -2.575376 \\
\hline $\mathrm{O}$ & -0.563237 & 2.192731 & -3.347809 \\
\hline $\mathrm{O}$ & -0.563237 & -2.192731 & -3.347809 \\
\hline $\mathrm{N}$ & -0.571247 & 1.462151 & 0.302109 \\
\hline $\mathrm{N}$ & -0.571247 & -1.462151 & 0.302109 \\
\hline $\mathrm{N}$ & 0.485576 & 1.816513 & 1.097237 \\
\hline $\mathrm{C}$ & 0.108123 & 2.776065 & 2.010103 \\
\hline $\mathrm{C}$ & -1.234979 & 3.030571 & 1.776310 \\
\hline $\mathrm{C}$ & -1.627506 & 2.195845 & 0.706187 \\
\hline $\mathrm{C}$ & 1.047205 & 3.361717 & 3.015022 \\
\hline $\mathrm{H}$ & -1.855985 & 3.731016 & 2.310400 \\
\hline $\mathrm{C}$ & -2.979028 & 2.089604 & 0.071644 \\
\hline $\mathrm{N}$ & 0.485576 & -1.816513 & 1.097237 \\
\hline $\mathrm{C}$ & 0.108123 & -2.776065 & 2.010103 \\
\hline $\mathrm{C}$ & -1.234979 & -3.030571 & 1.776310 \\
\hline $\mathrm{C}$ & -1.627506 & -2.195845 & 0.706187 \\
\hline C & 1.047205 & -3.361717 & 3.015022 \\
\hline $\mathrm{H}$ & -1.855985 & -3.731016 & 2.310400 \\
\hline $\mathrm{C}$ & -2.979028 & -2.089604 & 0.071644 \\
\hline $\mathrm{C}$ & 1.847753 & 1.233214 & 0.976017 \\
\hline $\mathrm{C}$ & 1.847753 & -1.233214 & 0.976017 \\
\hline $\mathrm{H}$ & 2.485476 & 1.976147 & 0.484071 \\
\hline $\mathrm{H}$ & 2.485476 & -1.976147 & 0.484071 \\
\hline $\mathrm{H}$ & 2.213932 & 1.102299 & 2.000431 \\
\hline $\mathrm{H}$ & 2.213932 & -1.102299 & 2.000431 \\
\hline $\mathrm{N}$ & 1.897012 & 0.000000 & 0.209983 \\
\hline $\mathrm{C}$ & 2.935630 & 0.000000 & -0.871928 \\
\hline $\mathrm{C}$ & 4.388381 & 0.000000 & -0.376269 \\
\hline $\mathrm{H}$ & 2.741241 & 0.876778 & -1.497087 \\
\hline $\mathrm{H}$ & 2.741241 & -0.876778 & -1.497087 \\
\hline $\mathrm{H}$ & 5.094269 & 0.000000 & -1.214288 \\
\hline $\mathrm{H}$ & 4.601544 & -0.887536 & 0.229863 \\
\hline $\mathrm{H}$ & 4.601544 & 0.887536 & 0.229863 \\
\hline $\mathrm{H}$ & 1.907781 & 3.834052 & 2.528995 \\
\hline $\mathrm{H}$ & 0.552943 & 4.125393 & 3.625281 \\
\hline $\mathrm{H}$ & 1.432699 & 2.594566 & 3.695305 \\
\hline $\mathrm{H}$ & 1.907781 & -3.834052 & 2.528995 \\
\hline $\mathrm{H}$ & 0.552943 & -4.125393 & 3.625281 \\
\hline $\mathrm{H}$ & 1.432699 & -2.594566 & 3.695305 \\
\hline $\mathrm{H}$ & -3.275856 & 3.040669 & -0.382131 \\
\hline $\mathrm{H}$ & -2.985655 & 1.326390 & -0.713215 \\
\hline $\mathrm{H}$ & -3.739761 & 1.818099 & 0.810639 \\
\hline $\mathrm{H}$ & -3.275856 & -3.040669 & -0.382131 \\
\hline $\mathrm{H}$ & -2.985655 & -1.326390 & -0.713215 \\
\hline $\mathrm{H}$ & -3.739761 & -1.818099 & 0.810639 \\
\hline
\end{tabular}


Table S8. Optimized structure of $\left[\mathrm{Rh}\left(\square^{2}-\mathrm{N}_{3}{ }^{M e}\right)(\mathrm{CO})_{2}\right]^{+}$

\begin{tabular}{|c|c|c|c|}
\hline $\mathrm{Rh}$ & -0.579356 & 0.000000 & -1.480625 \\
\hline $\mathrm{C}$ & -0.565591 & 1.365035 & -2.794966 \\
\hline $\mathrm{C}$ & -0.565591 & -1.365035 & -2.794966 \\
\hline $\mathrm{O}$ & -0.551356 & 2.209507 & -3.566030 \\
\hline $\mathrm{O}$ & -0.551356 & -2.209507 & -3.566030 \\
\hline $\mathrm{N}$ & -0.602518 & 1.484064 & 0.054225 \\
\hline $\mathrm{N}$ & -0.602518 & -1.484064 & 0.054225 \\
\hline $\mathrm{N}$ & 0.509002 & 1.860173 & 0.766868 \\
\hline $\mathrm{C}$ & 0.182624 & 2.833988 & 1.683871 \\
\hline $\mathrm{C}$ & -1.176474 & 3.072502 & 1.537947 \\
\hline $\mathrm{C}$ & -1.636239 & 2.214668 & 0.513704 \\
\hline C & 1.169738 & 3.457288 & 2.617247 \\
\hline $\mathrm{H}$ & -1.765993 & 3.778101 & 2.100121 \\
\hline C & -3.025802 & 2.082339 & -0.026254 \\
\hline $\mathrm{N}$ & 0.509002 & -1.860173 & 0.766868 \\
\hline C & 0.182624 & -2.833988 & 1.683871 \\
\hline $\mathrm{C}$ & -1.176474 & -3.072502 & 1.537947 \\
\hline C & -1.636239 & -2.214668 & 0.513704 \\
\hline $\mathrm{C}$ & 1.169738 & -3.457288 & 2.617247 \\
\hline $\mathrm{H}$ & -1.765993 & -3.778101 & 2.100121 \\
\hline $\mathrm{C}$ & -3.025802 & -2.082339 & -0.026254 \\
\hline $\mathrm{C}$ & 1.828861 & 1.237608 & 0.502496 \\
\hline $\mathrm{C}$ & 1.828861 & -1.237608 & 0.502496 \\
\hline $\mathrm{H}$ & 2.578513 & 1.983851 & 0.782489 \\
\hline $\mathrm{H}$ & 2.578513 & -1.983851 & 0.782489 \\
\hline $\mathrm{H}$ & 1.875050 & 1.096842 & -0.581620 \\
\hline $\mathrm{H}$ & 1.875050 & -1.096842 & -0.581620 \\
\hline $\mathrm{N}$ & 2.074771 & 0.000000 & 1.203364 \\
\hline C & 3.115436 & 0.000000 & 2.259556 \\
\hline C & 4.560093 & 0.000000 & 1.735996 \\
\hline $\mathrm{H}$ & 2.942557 & -0.874062 & 2.896607 \\
\hline $\mathrm{H}$ & 2.942557 & 0.874062 & 2.896607 \\
\hline $\mathrm{H}$ & 5.280295 & 0.000000 & 2.561959 \\
\hline $\mathrm{H}$ & 4.758634 & 0.886092 & 1.122540 \\
\hline $\mathrm{H}$ & 4.758634 & -0.886092 & 1.122540 \\
\hline $\mathrm{H}$ & 2.023423 & 3.873920 & 2.072728 \\
\hline $\mathrm{H}$ & 0.714700 & 4.270715 & 3.191823 \\
\hline $\mathrm{H}$ & 1.558626 & 2.724508 & 3.331852 \\
\hline $\mathrm{H}$ & 2.023423 & -3.873920 & 2.072728 \\
\hline $\mathrm{H}$ & 0.714700 & -4.270715 & 3.191823 \\
\hline $\mathrm{H}$ & 1.558626 & -2.724508 & 3.331852 \\
\hline $\mathrm{H}$ & -3.446146 & 3.061691 & -0.277486 \\
\hline $\mathrm{H}$ & -3.039556 & 1.469695 & -0.933676 \\
\hline $\mathrm{H}$ & -3.690015 & 1.611039 & 0.705616 \\
\hline $\mathrm{H}$ & -3.446146 & -3.061691 & -0.277486 \\
\hline $\mathrm{H}$ & -3.039556 & -1.469695 & -0.933676 \\
\hline $\mathrm{H}$ & -3.690015 & -1.611039 & 0.705616 \\
\hline
\end{tabular}


Table S9. Optimized structure of $\left[\mathrm{Rh}\left(\square^{3}-\mathrm{N}_{3}{ }^{H}\right)(\mathrm{CO})\right]^{+}$

$\begin{array}{rrrr}\mathrm{Rh} & 0.003332 & 0.000000 & -0.825917 \\ \mathrm{C} & 0.003332 & 0.000000 & -2.692189 \\ \mathrm{O} & -0.004665 & 0.000000 & -3.841332 \\ \mathrm{~N} & -0.011694 & 2.024289 & -0.497132 \\ \mathrm{~N} & -0.011694 & -2.024289 & -0.497132 \\ \mathrm{~N} & -0.455094 & 2.319196 & 0.769555 \\ \mathrm{C} & -0.459629 & 3.676101 & 0.974650 \\ \mathrm{C} & -0.006358 & 4.263494 & -0.194092 \\ \mathrm{C} & 0.260786 & 3.198582 & -1.083170 \\ \mathrm{H} & -0.775520 & 4.110366 & 1.908888 \\ \mathrm{H} & 0.116721 & 5.316935 & -0.384584 \\ \mathrm{H} & 0.628930 & 3.232185 & -2.095353 \\ \mathrm{~N} & -0.455094 & -2.319196 & 0.769555 \\ \mathrm{C} & -0.459629 & -3.676101 & 0.974650 \\ \mathrm{C} & -0.006358 & -4.263494 & -0.194092 \\ \mathrm{C} & 0.260786 & -3.198582 & -1.083170 \\ \mathrm{H} & -0.775520 & -4.110366 & 1.908888 \\ \mathrm{H} & 0.116721 & -5.316935 & -0.384584 \\ \mathrm{H} & 0.628930 & -3.232185 & -2.095353 \\ \mathrm{C} & -0.841389 & 1.238551 & 1.683286 \\ \mathrm{C} & -0.841389 & -1.238551 & 1.683286 \\ \mathrm{H} & -0.665871 & 1.585281 & 2.705274 \\ \mathrm{H} & -0.665871 & -1.585281 & 2.705274 \\ \mathrm{H} & -1.905263 & 1.019724 & 1.557689 \\ \mathrm{H} & -1.905263 & -1.019724 & 1.557689 \\ \mathrm{~N} & -0.074003 & 0.000000 & 1.353888 \\ \mathrm{C} & 1.336535 & 0.000000 & 1.922553 \\ \mathrm{C} & 1.433288 & 0.000000 & 3.452597 \\ \mathrm{H} & 1.831040 & 0.877843 & 1.498548 \\ \mathrm{H} & 1.831040 & -0.877843 & 1.498548 \\ \mathrm{H} & 2.474929 & 0.000000 & 3.789250 \\ \mathrm{H} & 0.962832 & -0.888912 & 3.884869 \\ \mathrm{H} & 0.962832 & 0.888912 & 3.884869\end{array}$


Table S10. Optimized structure of $\left[\mathrm{Rh}\left(\square^{3}-\mathrm{N}_{3}{ }^{M e}\right)(\mathrm{CO})\right]^{+}$

\begin{tabular}{|c|c|c|c|}
\hline $\mathrm{Rh}$ & 1.000000 & 0.000000 & 1.000000 \\
\hline $\mathrm{C}$ & 1.000000 & 0.000000 & -0.859296 \\
\hline $\mathrm{O}$ & 0.971266 & 0.000000 & -2.010326 \\
\hline $\mathrm{N}$ & 0.989955 & 2.030236 & 1.337721 \\
\hline $\mathrm{N}$ & 0.989955 & -2.030236 & 1.337721 \\
\hline $\mathrm{N}$ & 0.493423 & 2.323114 & 2.594133 \\
\hline C & 0.488415 & 3.684044 & 2.816308 \\
\hline $\mathrm{C}$ & 0.998130 & 4.257777 & 1.661437 \\
\hline C & 1.300050 & 3.204931 & 0.762514 \\
\hline $\mathrm{C}$ & 0.006600 & 4.305054 & 4.087442 \\
\hline $\mathrm{H}$ & 1.138388 & 5.310952 & 1.481776 \\
\hline $\mathrm{C}$ & 1.871217 & 3.296503 & -0.616649 \\
\hline $\mathrm{N}$ & 0.493423 & -2.323114 & 2.594133 \\
\hline C & 0.488415 & -3.684044 & 2.816308 \\
\hline $\mathrm{C}$ & 0.998130 & -4.257777 & 1.661437 \\
\hline C & 1.300050 & -3.204931 & 0.762514 \\
\hline $\mathrm{C}$ & 0.006600 & -4.305054 & 4.087442 \\
\hline $\mathrm{H}$ & 1.138388 & -5.310952 & 1.481776 \\
\hline C & 1.871217 & -3.296503 & -0.616649 \\
\hline C & 0.063986 & 1.237258 & 3.474166 \\
\hline $\mathrm{C}$ & 0.063986 & -1.237258 & 3.474166 \\
\hline $\mathrm{H}$ & 0.185313 & 1.568527 & 4.509405 \\
\hline $\mathrm{H}$ & 0.185313 & -1.568527 & 4.509405 \\
\hline $\mathrm{H}$ & -0.991678 & 1.013167 & 3.296588 \\
\hline $\mathrm{H}$ & -0.991678 & -1.013167 & 3.296588 \\
\hline $\mathrm{N}$ & 0.846325 & 0.000000 & $3.17358 \varepsilon$ \\
\hline C & 2.231530 & 0.000000 & 3.794579 \\
\hline C & 2.273101 & 0.000000 & 5.327572 \\
\hline $\mathrm{H}$ & 2.741635 & 0.878096 & 3.389970 \\
\hline $\mathrm{H}$ & 2.741635 & -0.878096 & 3.38997 \\
\hline $\mathrm{H}$ & 3.302030 & 0.000000 & 5.701417 \\
\hline $\mathrm{H}$ & 1.786434 & -0.888500 & 5.742517 \\
\hline $\mathrm{H}$ & 1.786434 & 0.888500 & 5.742517 \\
\hline $\mathrm{H}$ & 0.574974 & 3.939847 & 4.949696 \\
\hline $\mathrm{H}$ & 0.109053 & 5.395358 & 4.062005 \\
\hline $\mathrm{H}$ & -1.049530 & 4.076573 & 4.267141 \\
\hline $\mathrm{H}$ & 0.574974 & -3.939847 & 4.949696 \\
\hline $\mathrm{H}$ & 0.109053 & -5.395358 & 4.062005 \\
\hline $\mathrm{H}$ & -1.049530 & -4.076573 & 4.26714 \\
\hline $\mathrm{H}$ & 2.274692 & 4.295395 & -0.80896 \\
\hline $\mathrm{H}$ & 2.681925 & 2.574497 & -0.754574 \\
\hline $\mathrm{H}$ & 1.106845 & 3.091732 & -1.372536 \\
\hline $\mathrm{H}$ & 2.274692 & -4.295395 & -0.80896 \\
\hline $\mathrm{H}$ & 2.681925 & -2.574497 & -0.75457 \\
\hline $\mathrm{H}$ & 1.106845 & -3.091732 & -1.372536 \\
\hline
\end{tabular}


Table S11. Transition State for the $\left[\mathrm{Rh}\left(\mathrm{N}_{3}{ }^{M e}\right)(\mathrm{cod})\right]^{+}$complex

\begin{tabular}{|c|c|c|c|}
\hline $\mathrm{Rh}$ & 0.991647 & 0.029334 & 0.834201 \\
\hline $\mathrm{X}$ & 1.105496 & 1.450268 & -0.677753 \\
\hline $\mathrm{x}$ & 0.985498 & -1.401829 & -0.672231 \\
\hline C & 0.413082 & 1.428112 & -0.768529 \\
\hline C & 1.797910 & 1.472424 & -0.586977 \\
\hline $\mathrm{C}$ & 0.288387 & -1.426864 & -0.626440 \\
\hline $\mathrm{C}$ & 1.682608 & -1.376793 & -0.718022 \\
\hline $\mathrm{H}$ & -0.165330 & 2.198720 & -0.262687 \\
\hline $\mathrm{H}$ & 2.179887 & 2.283752 & 0.032032 \\
\hline $\mathrm{H}$ & -0.131202 & -2.244890 & -0.041613 \\
\hline $\mathrm{H}$ & 2.228595 & -2.145755 & -0.173171 \\
\hline C & -0.298186 & 0.714431 & -1.914326 \\
\hline C & 2.843809 & 0.783862 & -1.443514 \\
\hline $\mathrm{C}$ & -0.696673 & -0.742296 & -1.556095 \\
\hline $\mathrm{C}$ & 2.463709 & -0.671476 & -1.822342 \\
\hline $\mathrm{H}$ & 0.334924 & 0.729258 & -2.807333 \\
\hline $\mathrm{H}$ & -1.201857 & 1.276191 & -2.175248 \\
\hline $\mathrm{H}$ & 3.038831 & 1.375350 & -2.351572 \\
\hline $\mathrm{H}$ & 3.782306 & 0.773348 & -0.876781 \\
\hline $\mathrm{H}$ & -0.826495 & -1.337273 & -2.473416 \\
\hline $\mathrm{H}$ & -1.674907 & -0.735144 & -1.059805 \\
\hline $\mathrm{H}$ & 1.884479 & -0.687466 & -2.751348 \\
\hline $\mathrm{H}$ & 3.379242 & -1.237607 & -2.026898 \\
\hline $\mathrm{N}$ & 0.896193 & 1.450338 & 2.468907 \\
\hline $\mathrm{N}$ & 0.947563 & -1.371620 & 2.489412 \\
\hline $\mathrm{N}$ & 1.883825 & 1.442419 & 3.433434 \\
\hline $\mathrm{C}$ & 1.380703 & 1.869023 & 4.648305 \\
\hline $\mathrm{C}$ & 0.035214 & 2.125933 & 4.445913 \\
\hline C & -0.231823 & 1.854534 & 3.083752 \\
\hline C & 2.210303 & 2.040034 & 5.879659 \\
\hline $\mathrm{H}$ & -0.668615 & 2.474664 & 5.184000 \\
\hline C & -1.543760 & 1.997315 & 2.374151 \\
\hline $\mathrm{N}$ & 2.051898 & -1.463371 & 3.305027 \\
\hline $\mathrm{C}$ & 1.713606 & -1.998339 & 4.532173 \\
\hline C & 0.348881 & -2.228440 & 4.490863 \\
\hline $\mathrm{C}$ & -0.095428 & -1.823617 & 3.207617 \\
\hline $\mathrm{C}$ & 2.709594 & -2.283005 & 5.609321 \\
\hline $\mathrm{H}$ & -0.255021 & -2.646890 & 5.279659 \\
\hline $\mathrm{C}$ & -1.491775 & -1.868201 & 2.669525 \\
\hline C & 3.298871 & 1.397902 & 3.062695 \\
\hline $\mathrm{C}$ & 3.388403 & -1.243911 & 2.764217 \\
\hline $\mathrm{H}$ & 3.413949 & 2.094655 & 2.224207 \\
\hline $\mathrm{H}$ & 3.368857 & -1.667298 & 1.753839 \\
\hline $\mathrm{H}$ & 3.837068 & 1.833824 & 3.916228 \\
\hline $\mathrm{H}$ & 4.059797 & -1.864598 & 3.367110 \\
\hline $\mathrm{N}$ & 3.938589 & 0.122504 & 2.707185 \\
\hline C & 5.398515 & 0.179376 & 2.935158 \\
\hline C & 6.128444 & 1.154851 & 2.000458 \\
\hline $\mathrm{H}$ & 5.800711 & -0.824755 & 2.765068 \\
\hline $\mathrm{H}$ & 5.620354 & 0.441480 & 3.988208 \\
\hline $\mathrm{H}$ & 7.208923 & 1.097583 & 2.169840 \\
\hline $\mathrm{H}$ & 5.828184 & 2.194975 & 2.166674 \\
\hline $\mathrm{H}$ & 5.930229 & 0.902708 & 0.953268 \\
\hline $\mathrm{H}$ & 2.908864 & 2.882274 & 5.794469 \\
\hline $\mathrm{H}$ & 1.558395 & 2.244661 & 6.731870 \\
\hline $\mathrm{H}$ & 2.795676 & 1.144262 & 6.114669 \\
\hline $\mathrm{H}$ & 3.397316 & -3.091526 & 5.329721 \\
\hline $\mathrm{H}$ & 2.189034 & -2.598388 & 6.516206 \\
\hline $\mathrm{H}$ & 3.316291 & -1.405133 & 5.859472 \\
\hline $\mathrm{H}$ & -1.679923 & 3.021066 & 2.003343 \\
\hline $\mathrm{H}$ & -1.615998 & 1.317057 & 1.522304 \\
\hline $\mathrm{H}$ & -2.373363 & 1.786681 & 3.054997 \\
\hline $\mathrm{H}$ & -1.821843 & -2.902569 & 2.516304 \\
\hline $\mathrm{H}$ & -1.563573 & -1.340922 & 1.715714 \\
\hline $\mathrm{H}$ & -2.194730 & -1.404896 & 3.369814 \\
\hline
\end{tabular}


Table S12. Transition State for the $\left[\mathrm{Rh}\left(\mathrm{N}_{3} M e\right)(\mathrm{CO})_{2}\right]^{+}$complex

\begin{tabular}{|c|c|c|c|}
\hline $\mathrm{Rh}$ & -0.299340 & 0.087517 & -1.440029 \\
\hline $\mathrm{C}$ & -0.181075 & 1.555833 & -2.628712 \\
\hline C & 0.011105 & -1.158131 & -2.831716 \\
\hline 0 & -0.112020 & 2.467094 & -3.316798 \\
\hline 0 & 0.201812 & -1.938336 & -3.646238 \\
\hline $\mathrm{N}$ & -0.677973 & 1.349772 & 0.236100 \\
\hline $\mathrm{N}$ & -0.490023 & -1.449153 & 0.027378 \\
\hline $\mathrm{N}$ & 0.230197 & 1.407999 & 1.269414 \\
\hline C & -0.390024 & 1.830816 & 2.428276 \\
\hline $\mathrm{C}$ & -1.726667 & 2.018100 & 2.113225 \\
\hline $\mathrm{C}$ & -1.872829 & 1.709983 & 0.740331 \\
\hline $\mathrm{C}$ & 0.334295 & 2.060124 & 3.714914 \\
\hline $\mathrm{H}$ & -2.502297 & 2.348303 & 2.784674 \\
\hline C & -3.119025 & 1.759733 & -0.086682 \\
\hline $\mathrm{N}$ & 0.418336 & -1.534796 & 1.059096 \\
\hline C & -0.139909 & -2.199194 & 2.133271 \\
\hline $\mathrm{C}$ & -1.439386 & -2.514821 & 1.768849 \\
\hline C & -1.625667 & -2.033586 & 0.451947 \\
\hline C & 0.605953 & -2.513590 & 3.389252 \\
\hline $\mathrm{H}$ & -2.163667 & -3.037889 & 2.371591 \\
\hline C & -2.851026 & -2.134997 & -0.401107 \\
\hline $\mathrm{C}$ & 1.666677 & 1.382153 & 1.007265 \\
\hline $\mathrm{C}$ & 1.840241 & -1.286872 & 0.830672 \\
\hline $\mathrm{H}$ & 1.832444 & 2.010730 & 0.121073 \\
\hline $\mathrm{H}$ & 2.090627 & -1.770102 & -0.124398 \\
\hline $\mathrm{H}$ & 2.130006 & 1.904585 & 1.855471 \\
\hline $\mathrm{H}$ & 2.364449 & -1.849513 & 1.615228 \\
\hline $\mathrm{N}$ & 2.322046 & 0.091979 & 0.811465 \\
\hline C & 3.709952 & 0.214642 & 0.336401 \\
\hline $\mathrm{C}$ & 3.868055 & 0.345027 & -1.189247 \\
\hline $\mathrm{H}$ & 4.271140 & -0.658495 & 0.695963 \\
\hline $\mathrm{H}$ & 4.164627 & 1.082676 & 0.833402 \\
\hline $\mathrm{H}$ & 4.926887 & 0.431187 & -1.456790 \\
\hline $\mathrm{H}$ & 3.355053 & 1.235229 & -1.569869 \\
\hline $\mathrm{H}$ & 3.462730 & -0.530055 & -1.709295 \\
\hline $\mathrm{H}$ & 0.962725 & 2.958930 & 3.677038 \\
\hline $\mathrm{H}$ & -0.388675 & 2.202435 & 4.521071 \\
\hline $\mathrm{H}$ & 0.977502 & 1.215373 & 3.984042 \\
\hline $\mathrm{H}$ & 1.351797 & -3.304003 & 3.237198 \\
\hline $\mathrm{H}$ & -0.093351 & -2.868216 & 4.149573 \\
\hline $\mathrm{H}$ & 1.125888 & -1.637901 & 3.792321 \\
\hline $\mathrm{H}$ & -3.556157 & 2.764002 & -0.063962 \\
\hline $\mathrm{H}$ & -2.918272 & 1.498054 & -1.12870 \\
\hline $\mathrm{H}$ & -3.876889 & 1.067930 & 0.297068 \\
\hline $\mathrm{H}$ & -3.723086 & -1.711492 & 0.108253 \\
\hline $\mathrm{H}$ & -3.082288 & -3.183492 & -0.621153 \\
\hline $\mathrm{H}$ & -2.721323 & -1.608494 & -1.350025 \\
\hline
\end{tabular}


Table S13. Optimized structure of $\left[\mathrm{Rh}\left(\square^{3}-\mathrm{Tp} \mathrm{p}^{H}\right)(\mathrm{CO})\right]^{+}$(SPy)

$\begin{array}{rrrr}\mathrm{Rh} & -0.622771 & 0.000000 & -1.089654 \\ \mathrm{C} & -0.736750 & 1.342529 & -2.393713 \\ \mathrm{C} & -0.736750 & -1.342529 & -2.393713 \\ \mathrm{O} & -0.819001 & 2.197654 & -3.159151 \\ \mathrm{O} & -0.819001 & -2.197654 & -3.159151 \\ \mathrm{~N} & -0.784209 & 1.457803 & 0.463304 \\ \mathrm{~N} & -0.784209 & -1.457803 & 0.463304 \\ \mathrm{~N} & -0.179936 & 1.261057 & 1.669339 \\ \mathrm{C} & -0.427944 & 2.326346 & 2.488129 \\ \mathrm{C} & -1.213918 & 3.235118 & 1.790637 \\ \mathrm{C} & -1.410449 & 2.645537 & 0.526265 \\ \mathrm{H} & -0.032044 & 2.356236 & 3.490177 \\ \mathrm{H} & -1.589695 & 4.182441 & 2.141496 \\ \mathrm{H} & -1.964024 & 3.020068 & -0.319319 \\ \mathrm{~N} & -0.179936 & -1.261057 & 1.669339 \\ \mathrm{C} & -0.427944 & -2.326346 & 2.488129 \\ \mathrm{C} & -1.213918 & -3.235118 & 1.790637 \\ \mathrm{C} & -1.410449 & -2.645537 & 0.526265 \\ \mathrm{H} & -0.032044 & -2.356236 & 3.490177 \\ \mathrm{H} & -1.589695 & -4.182441 & 2.141496 \\ \mathrm{H} & -1.964024 & -3.020068 & -0.319319 \\ \mathrm{~N} & 1.937852 & 0.000000 & -0.192974 \\ \mathrm{~N} & 1.972162 & 0.000000 & 1.169817 \\ \mathrm{C} & 3.269632 & 0.000000 & 1.597996 \\ \mathrm{C} & 4.094719 & 0.000000 & 0.480254 \\ \mathrm{C} & 3.212910 & 0.000000 & -0.618509 \\ \mathrm{H} & 3.503644 & 0.000000 & 2.650122 \\ \mathrm{H} & 5.172370 & 0.000000 & 0.460236 \\ \mathrm{H} & 3.440304 & 0.000000 & -1.672085 \\ \mathrm{~B} & 0.675676 & 0.000000 & 1.993031 \\ \mathrm{H} & 0.938655 & 0.000000 & 3.163541\end{array}$


Table S14. Optimized structure of $\left[\mathrm{Rh}\left(\square^{2}-\mathrm{Tp}{ }^{H}\right)(\mathrm{CO})\right]^{+}(\mathrm{SP} 1)$

$\begin{array}{rrrr}\mathrm{Rh} & -0.968296 & 0.010039 & -0.800162 \\ \mathrm{C} & -1.238392 & 1.367318 & -2.080309 \\ \mathrm{C} & -1.238392 & -1.347240 & -2.080309 \\ \mathrm{O} & -1.392745 & 2.215567 & -2.838187 \\ \mathrm{O} & -1.392745 & -2.195489 & -2.838187 \\ \mathrm{~N} & -0.649301 & 1.462260 & 0.696027 \\ \mathrm{~N} & -0.649301 & -1.442182 & 0.696027 \\ \mathrm{~N} & 0.302551 & 1.271615 & 1.653017 \\ \mathrm{C} & 0.312947 & 2.341321 & 2.494446 \\ \mathrm{C} & -0.656234 & 3.246025 & 2.070966 \\ \mathrm{C} & -1.235683 & 2.651968 & 0.937824 \\ \mathrm{H} & 1.001437 & 2.378502 & 3.322394 \\ \mathrm{H} & -0.906680 & 4.194820 & 2.515896 \\ \mathrm{H} & -2.026337 & 3.018232 & 0.304093 \\ \mathrm{~N} & 0.302551 & -1.251537 & 1.653017 \\ \mathrm{C} & 0.312947 & -2.321243 & 2.494446 \\ \mathrm{C} & -0.656234 & -3.225947 & 2.070966 \\ \mathrm{C} & -1.235683 & -2.631890 & 0.937824 \\ \mathrm{H} & 1.001437 & -2.358424 & 3.322394 \\ \mathrm{H} & -0.906680 & -4.174742 & 2.515896 \\ \mathrm{H} & -2.026337 & -2.998154 & 0.304093 \\ \mathrm{~B} & 1.213943 & 0.010039 & 1.694627 \\ \mathrm{H} & 1.791605 & 0.010039 & 2.750869 \\ \mathrm{~N} & 2.153405 & 0.010039 & 0.472256 \\ \mathrm{~N} & 2.618562 & -1.168719 & -0.039760 \\ \mathrm{C} & 3.394146 & -0.828794 & -1.075785 \\ \mathrm{C} & 3.444481 & 0.576831 & -1.252439 \\ \mathrm{C} & 2.638893 & 1.081290 & -0.244150 \\ \mathrm{H} & 3.886188 & -1.594538 & -1.655444 \\ \mathrm{H} & 3.985435 & 1.135638 & -2.000487 \\ \mathrm{H} & 2.381455 & 2.097848 & 0.010422\end{array}$


Table S15. Optimized structure of $\left[\mathrm{Rh}\left(\square^{2}-\mathrm{Tp}{ }^{H}\right)(\mathrm{CO})\right]^{+}(\mathrm{SP} 2)$

$\begin{array}{rrrr}\mathrm{Rh} & -0.935127 & 0.000000 & -1.593826 \\ \mathrm{C} & -1.138681 & 1.361859 & -2.883056 \\ \mathrm{C} & -1.138681 & -1.361859 & -2.883056 \\ \mathrm{O} & -1.255682 & 2.212886 & -3.644766 \\ \mathrm{O} & -1.255682 & -2.212886 & -3.644766 \\ \mathrm{~N} & -0.692595 & 1.444735 & -0.079587 \\ \mathrm{~N} & -0.692595 & -1.444735 & -0.079587 \\ \mathrm{~N} & 0.234249 & 1.258078 & 0.901322 \\ \mathrm{C} & 0.205452 & 2.315172 & 1.756011 \\ \mathrm{C} & -0.768375 & 3.209973 & 1.313652 \\ \mathrm{C} & -1.306875 & 2.623670 & 0.158776 \\ \mathrm{H} & 0.871047 & 2.349652 & 2.602325 \\ \mathrm{H} & -1.045115 & 4.148562 & 1.764115 \\ \mathrm{H} & -2.084940 & 2.983651 & -0.493513 \\ \mathrm{~N} & 0.234249 & -1.258078 & 0.901322 \\ \mathrm{C} & 0.205452 & -2.315172 & 1.756011 \\ \mathrm{C} & -0.768375 & -3.209973 & 1.313652 \\ \mathrm{C} & -1.306875 & -2.623670 & 0.158776 \\ \mathrm{H} & 0.871047 & -2.349652 & 2.602325 \\ \mathrm{H} & -1.045115 & -4.148562 & 1.764115 \\ \mathrm{H} & -2.084940 & -2.983651 & -0.493513 \\ \mathrm{~B} & 1.172893 & 0.000000 & 0.861996 \\ \mathrm{H} & 1.776928 & 0.000000 & -0.180494 \\ \mathrm{~N} & 2.150155 & 0.000000 & 2.020715 \\ \mathrm{~N} & 1.739312 & 0.000000 & 3.331965 \\ \mathrm{C} & 2.863716 & 0.000000 & 4.056441 \\ \mathrm{C} & 4.019171 & 0.000000 & 3.233179 \\ \mathrm{C} & 3.525119 & 0.000000 & 1.941095 \\ \mathrm{H} & 2.811517 & 0.000000 & 5.134028 \\ \mathrm{H} & 5.053263 & 0.000000 & 3.540703 \\ \mathrm{H} & 4.027668 & 0.000000 & 0.986441\end{array}$


Table S16. Optimized structure of $\left[\mathrm{Rh}\left(\square^{3}-\mathrm{Tp}^{M e}\right)(\mathrm{CO})\right]^{+}(\mathrm{SPy})$

\begin{tabular}{|c|c|c|c|}
\hline $\mathrm{Rh}$ & 1.000000 & 0.000000 & 1.000000 \\
\hline $\mathrm{C}$ & 1.028325 & 1.324745 & -0.325635 \\
\hline $\mathrm{C}$ & 1.028325 & -1.324745 & -0.325635 \\
\hline $\mathrm{O}$ & 1.073177 & 2.149883 & -1.128273 \\
\hline 0 & 1.073177 & -2.149883 & -1.128273 \\
\hline $\mathrm{N}$ & 0.710095 & 1.436133 & 2.563948 \\
\hline $\mathrm{N}$ & 0.710095 & -1.436133 & 2.563948 \\
\hline $\mathrm{N}$ & 1.376785 & 1.264402 & 3.752829 \\
\hline $\mathrm{C}$ & 1.051116 & 2.287315 & 4.607331 \\
\hline $\mathrm{C}$ & 0.159128 & 3.120748 & 3.939364 \\
\hline $\mathrm{C}$ & -0.032267 & 2.557668 & 2.662062 \\
\hline $\mathrm{C}$ & 1.596840 & 2.417517 & 5.994364 \\
\hline $\mathrm{H}$ & -0.295068 & 4.019608 & 4.324460 \\
\hline $\mathrm{C}$ & -0.901162 & 3.071386 & 1.555698 \\
\hline $\mathrm{N}$ & 1.376785 & -1.264402 & 3.752829 \\
\hline $\mathrm{C}$ & 1.051116 & -2.287315 & 4.607331 \\
\hline $\mathrm{C}$ & 0.159128 & -3.120748 & 3.939364 \\
\hline $\mathrm{C}$ & -0.032267 & -2.557668 & 2.662062 \\
\hline $\mathrm{C}$ & 1.596840 & -2.417517 & 5.994364 \\
\hline $\mathrm{H}$ & -0.295068 & -4.019608 & 4.324460 \\
\hline $\mathrm{C}$ & -0.901162 & -3.071386 & 1.555698 \\
\hline $\mathrm{H}$ & 2.691559 & 2.438905 & 5.987672 \\
\hline $\mathrm{H}$ & 1.247883 & 3.339369 & 6.471155 \\
\hline $\mathrm{H}$ & 1.283234 & 1.577384 & 6.622646 \\
\hline $\mathrm{H}$ & 2.691559 & -2.438905 & 5.987672 \\
\hline $\mathrm{H}$ & 1.247883 & -3.339369 & 6.471155 \\
\hline $\mathrm{H}$ & 1.283234 & -1.577384 & 6.622646 \\
\hline $\mathrm{H}$ & -0.321362 & 3.669182 & 0.844572 \\
\hline $\mathrm{H}$ & -1.362750 & 2.246974 & 1.002064 \\
\hline $\mathrm{H}$ & -1.705151 & 3.703454 & 1.947259 \\
\hline $\mathrm{H}$ & -0.321362 & -3.669182 & 0.844572 \\
\hline $\mathrm{H}$ & -1.362750 & -2.246974 & 1.002064 \\
\hline $\mathrm{H}$ & -1.705151 & -3.703454 & 1.947259 \\
\hline $\mathrm{N}$ & 3.460974 & 0.000000 & 1.882892 \\
\hline $\mathrm{N}$ & 3.549478 & 0.000000 & 3.247847 \\
\hline $\mathrm{C}$ & 4.871153 & 0.000000 & 3.644258 \\
\hline $\mathrm{C}$ & 5.634957 & 0.000000 & 2.480920 \\
\hline $\mathrm{C}$ & 4.713802 & 0.000000 & 1.406938 \\
\hline $\mathrm{C}$ & 5.336826 & 0.000000 & 5.069078 \\
\hline $\mathrm{H}$ & 6.712190 & 0.000000 & 2.415916 \\
\hline $\mathrm{C}$ & 4.998584 & 0.000000 & -0.066181 \\
\hline $\mathrm{H}$ & 4.490926 & 0.000000 & 5.764656 \\
\hline $\mathrm{H}$ & 5.945414 & -0.883461 & 5.289287 \\
\hline $\mathrm{H}$ & 5.945414 & 0.883461 & 5.289287 \\
\hline $\mathrm{H}$ & 6.076821 & 0.000000 & -0.261655 \\
\hline $\mathrm{H}$ & 4.572373 & -0.884675 & -0.552493 \\
\hline $\mathrm{H}$ & 4.572373 & 0.884675 & -0.552493 \\
\hline B & 2.242365 & 0.000000 & 4.060253 \\
\hline $\mathrm{H}$ & 2.484628 & 0.000000 & 5.227738 \\
\hline
\end{tabular}


Table S17. Optimized structure of $\left[\mathrm{Rh}\left(\square^{2}-\mathrm{Tp} \mathrm{p}^{M e}\right)(\mathrm{CO})\right]^{+}(\mathrm{SP} 2)$

\begin{tabular}{|c|c|c|c|}
\hline $\mathrm{Rh}$ & 1.000000 & 0.000000 & 1.000000 \\
\hline $\mathrm{C}$ & 0.811568 & 1.351149 & -0.299947 \\
\hline $\mathrm{C}$ & 0.811568 & -1.351149 & -0.299947 \\
\hline 0 & 0.720668 & 2.188935 & -1.081723 \\
\hline $\mathrm{O}$ & 0.720668 & -2.188935 & -1.081723 \\
\hline $\mathrm{N}$ & 1.236504 & 1.417479 & 2.544063 \\
\hline $\mathrm{N}$ & 1.236504 & -1.417479 & 2.544063 \\
\hline $\mathrm{N}$ & 2.294289 & 1.281065 & 3.418379 \\
\hline $\mathrm{C}$ & 2.242179 & 2.310267 & 4.327598 \\
\hline $\mathrm{C}$ & 1.132891 & 3.096675 & 4.009900 \\
\hline $\mathrm{C}$ & 0.526130 & 2.510035 & 2.889134 \\
\hline $\mathrm{C}$ & 3.198050 & 2.545905 & 5.451948 \\
\hline $\mathrm{H}$ & 0.812100 & 3.983669 & 4.532325 \\
\hline $\mathrm{C}$ & -0.697264 & 2.959521 & 2.149623 \\
\hline $\mathrm{N}$ & 2.294289 & -1.281065 & 3.418379 \\
\hline $\mathrm{C}$ & 2.242179 & -2.310267 & 4.327598 \\
\hline $\mathrm{C}$ & 1.132891 & -3.096675 & 4.009900 \\
\hline $\mathrm{C}$ & 0.526130 & -2.510035 & 2.889134 \\
\hline $\mathrm{C}$ & 3.198050 & -2.545905 & 5.451948 \\
\hline $\mathrm{H}$ & 0.812100 & -3.983669 & 4.532325 \\
\hline $\mathrm{C}$ & -0.697264 & -2.959521 & 2.149623 \\
\hline $\mathrm{H}$ & 4.203562 & 2.747652 & 5.073229 \\
\hline $\mathrm{H}$ & 2.886325 & 3.402205 & 6.055905 \\
\hline $\mathrm{H}$ & 3.252652 & 1.672349 & 6.107009 \\
\hline $\mathrm{H}$ & 4.203562 & -2.747652 & 5.073229 \\
\hline $\mathrm{H}$ & 2.886325 & -3.402205 & 6.055905 \\
\hline $\mathrm{H}$ & 3.252652 & -1.672349 & 6.107009 \\
\hline $\mathrm{H}$ & -0.430566 & 3.537351 & 1.258263 \\
\hline $\mathrm{H}$ & -1.299491 & 2.104351 & 1.824751 \\
\hline $\mathrm{H}$ & -1.330303 & 3.592724 & 2.780379 \\
\hline $\mathrm{H}$ & -0.430566 & -3.537351 & 1.258263 \\
\hline $\mathrm{H}$ & -1.299491 & -2.104351 & 1.824751 \\
\hline $\mathrm{H}$ & -1.330303 & -3.592724 & 2.780379 \\
\hline B & 3.208185 & 0.000000 & 3.153175 \\
\hline $\mathrm{H}$ & 3.399730 & 0.000000 & 1.965638 \\
\hline $\mathrm{N}$ & 4.555967 & 0.000000 & 3.848272 \\
\hline $\mathrm{N}$ & 4.706098 & 0.000000 & 5.217841 \\
\hline $\mathrm{C}$ & 6.018156 & 0.000000 & 5.457935 \\
\hline $\mathrm{C}$ & 6.749156 & 0.000000 & 4.237978 \\
\hline $\mathrm{C}$ & 5.801350 & 0.000000 & 3.229347 \\
\hline $\mathrm{C}$ & 6.550128 & 0.000000 & 6.860428 \\
\hline $\mathrm{H}$ & 7.820988 & 0.000000 & 4.112458 \\
\hline $\mathrm{C}$ & 6.007236 & 0.000000 & 1.746227 \\
\hline $\mathrm{H}$ & 5.734398 & 0.011280 & 7.592665 \\
\hline $\mathrm{H}$ & 7.159171 & -0.891060 & 7.052242 \\
\hline $\mathrm{H}$ & 7.177439 & 0.879780 & 7.045313 \\
\hline $\mathrm{H}$ & 7.072993 & -0.012505 & 1.493400 \\
\hline $\mathrm{H}$ & 5.545507 & -0.877846 & 1.281355 \\
\hline $\mathrm{H}$ & 5.566961 & 0.890351 & 1.284333 \\
\hline
\end{tabular}


Table C1. Summary of frequencies of each molecular geometry classified by metal (see scheme below). Compounds with $\mathrm{Epz}_{4}$ as Tp ligand are included in parenthesis.

\begin{tabular}{llll|llll}
\hline Geometry & Table & Rh & Other & Pd & Ir & Pt & Au \\
\hline SPy & C2 & 12 & 16 & 9 & 0 & 3 & 4 \\
TBP & C3 & $15(+2)$ & 9 & 2 & 5 & 2 & 0 \\
SP1 & C4 & $35(+2)$ & $45(+11)$ & $33(+9)$ & 1 & 10 & $1(+2)$ \\
SP2 & C5 & $3(+4)$ & $7(+11)$ & $5(+9)$ & 1 & 1 & $0(+2)$ \\
& & $65(+8)$ & $77(+22)$ & $49(+18)$ & 7 & 16 & $5(+4)$ \\
Data & 73 & 99 & 67 & 7 & 16 & 9 \\
Total & & 60 & 75 & 46 & 7 & 15 & 7 \\
Refcodes & & &
\end{tabular}

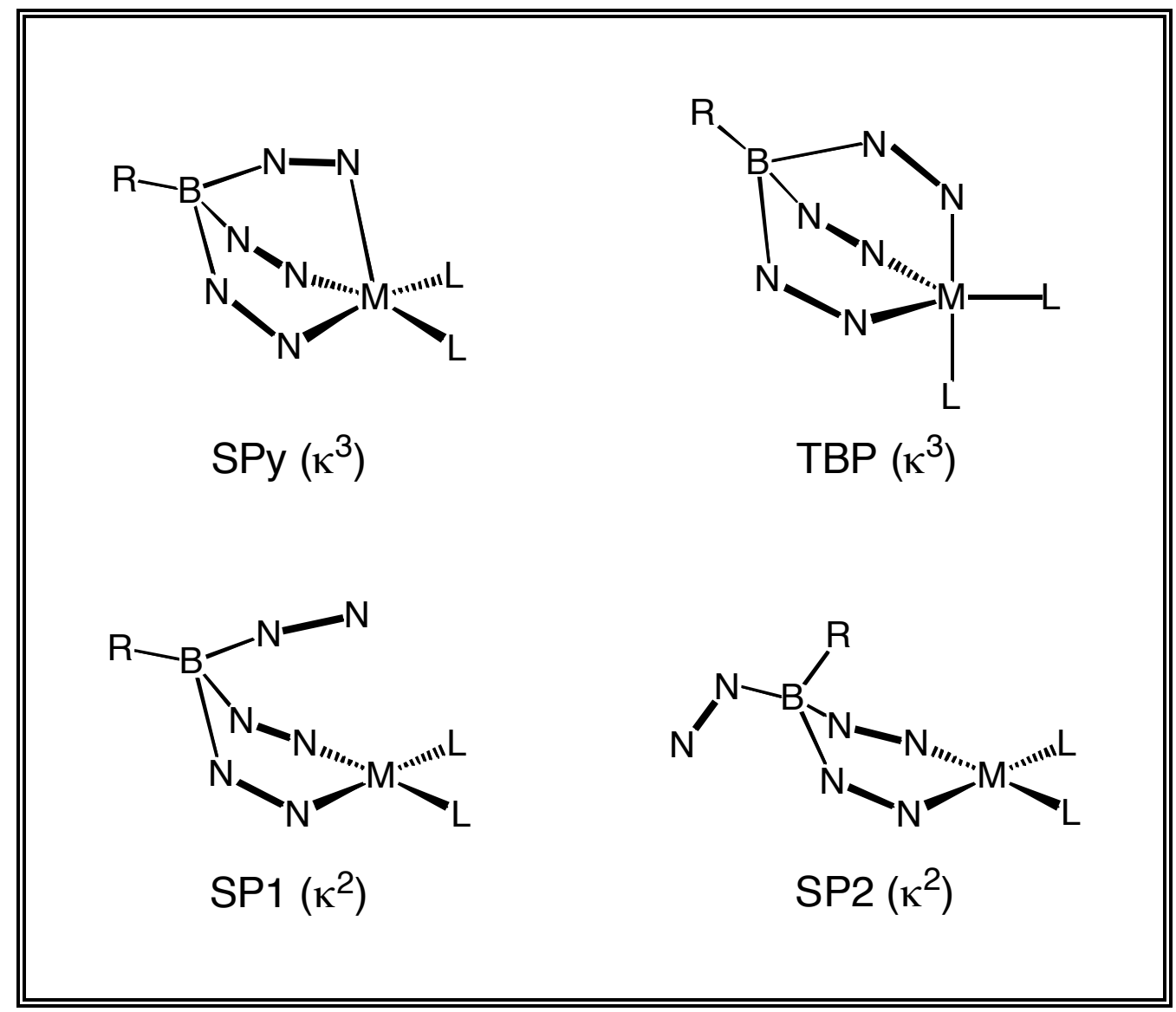


Table C2. Structural parameters and CSD refcodes for Tp compounds with structure of $\square^{3}$ squarepyramid (SPy).

\begin{tabular}{|c|c|c|c|c|}
\hline Metal & Refcode & $d\left(\mathrm{M}-\mathrm{L}_{a p}\right)$ & $d\left(\mathrm{M}-\mathrm{L}_{a p}\right)-d\left(\mathrm{M}-\mathrm{L}_{b s}\right)$ & $\square\left(\mathrm{M}-\mathrm{E}-\mathrm{C}-\mathrm{N}_{a p}\right)$ \\
\hline \multirow[t]{12}{*}{$\mathrm{Rh}$} & EFUGOB & 2.524 & +0.401 & 16.5 \\
\hline & EKADUP & 2.431 & +0.301 & 10.8 \\
\hline & " & 2.440 & +0.306 & 5.3 \\
\hline & IKANAJ & 2.426 & +0.303 & 1.5 \\
\hline & LIDKOY & 2.641 & +0.534 & 0.5 \\
\hline & " & 2.780 & +0.687 & 15.6 \\
\hline & QETNUY01 & 2.762 & +0.652 & 18.4 \\
\hline & QUHNAI & 2.893 & +0.775 & 17.5 \\
\hline & TIWWOL & 2.637 & +0.523 & 3.3 \\
\hline & RUWZIS & 2.835 & +0.744 & 25.9 \\
\hline & WAYMAK & 2.835 & +0.736 & 27.1 \\
\hline & WOGXIZ & 2.578 & +0.470 & 6.2 \\
\hline \multirow[t]{9}{*}{$\mathrm{Pd}$} & EJITEW & 2.925 & +0.903 & 0.4 \\
\hline & HAYMUP & 2.693 & +0.539 & 8.4 \\
\hline & QIYXAX & 2.784 & +0.799 & 21.5 \\
\hline & QUQTIF & 2.715 & +0.713 & 6.4 \\
\hline & QUQTOL & 2.794 & +0.783 & 3.2 \\
\hline & QUQVAZ & 3.003 & +0.965 & 15.3 \\
\hline & $\mathrm{UHEVUY}^{a}$ & 2.895 & +0.885 & 35.3 \\
\hline & UHEWAF $a$ & 2.899 & +0.885 & 1.4 \\
\hline & " & 3.019 & +0.991 & 35.2 \\
\hline \multirow[t]{3}{*}{$\mathrm{Pt}$} & QEHWOP & $3.165^{b}$ & +1.112 & 2.7 \\
\hline & TUYTEM & $3.154^{b}$ & +1.128 & 29.0 \\
\hline & VODCUM & $3.173^{b}$ & +1.113 & 1.3 \\
\hline \multirow[t]{4}{*}{$\mathrm{Au}$} & $\mathrm{BOFCEE}$ & 3.140 & +1.004 & 5.2 \\
\hline & CEPMAL & 3.312 & +1.189 & 27.1 \\
\hline & $\mathrm{DAYCAH}$ & 3.317 & +1.212 & 10.3 \\
\hline & FAGKOO & 3.157 & +1.143 & 6.0 \\
\hline \multicolumn{2}{|c|}{ Means Values } & 2.4 to 3.4 & +0.3 to +1.3 & $<36$ \\
\hline
\end{tabular}

$a$ Two or more different conformations for a REpz 3 as Tp ligand.

${ }^{b}$ A Pt $\cdots \mathrm{H}-\mathrm{N}$ interaction could exist. 
Table C3. Structural parameters and CSD refcodes for Tp compounds with structure of $\square^{3}$ trigonal bipyramid (TBP).

\begin{tabular}{|c|c|c|c|c|}
\hline Metal & Refcode & $d\left(\mathrm{M}-\mathrm{L}_{a p}\right)$ & $d\left(\mathrm{M}-\mathrm{L}_{a p}\right)-d\left(\mathrm{M}-\mathrm{L}_{b s}\right)$ & $\square\left(\mathrm{M}-\mathrm{E}-\mathrm{C}-\mathrm{N}_{a p}\right)$ \\
\hline \multirow[t]{17}{*}{$\mathrm{Rh}$} & $\mathrm{BIZVEL}^{a}$ & 2.167 & +0.076 & 3.4 \\
\hline & $"$ & 2.185 & +0.079 & 0.3 \\
\hline & EKAFEB & 2.167 & -0.026 & 4.4 \\
\hline & EKAGAY & 2.160 & -0.027 & 10.4 \\
\hline & FEGZIA & 2.196 & +0.014 & 3.2 \\
\hline & GAKQUE & 2.181 & +0.036 & 6.3 \\
\hline & GAWXUX & 2.293 & +0.124 & 2.8 \\
\hline & KASFIT & 2.227 & +0.015 & 0.7 \\
\hline & " & 2.232 & +0.033 & 10.2 \\
\hline & $\mathrm{NOHCAO}^{b}$ & 2.273 & +0.070 & 7.8 \\
\hline & PEMFOC & 2.166 & -0.039 & 1.7 \\
\hline & QOSRIZ & 2.218 & +0.077 & 2.9 \\
\hline & XAWQER & 2.258 & +0.094 & 0.3 \\
\hline & " & 2.288 & +0.127 & 0.2 \\
\hline & YIKHEF & 2.248 & +0.051 & 2.1 \\
\hline & $"$ & 2.264 & +0.077 & 7.9 \\
\hline & ZIPSOG & 2.242 & +0.055 & 7.7 \\
\hline \multirow[t]{2}{*}{$\mathrm{Pd}$} & XOQCIP & 2.186 & +0.112 & 3.0 \\
\hline & XOQCOV & 2.169 & +0.105 & 3.4 \\
\hline \multirow[t]{5}{*}{$\mathrm{Ir}$} & FEFXUJ & 2.184 & +0.019 & 10.9 \\
\hline & NAKRAS & 2.170 & +0.010 & 5.2 \\
\hline & NULNIR & 2.241 & +0.088 & 0.5 \\
\hline & QIPLIK & 2.202 & +0.058 & 3.7 \\
\hline & UHIRIM & 2.120 & -0.020 & 0.0 \\
\hline \multirow[t]{2}{*}{$\mathrm{Pt}$} & MBFYPT & 2.150 & +0.032 & 0.0 \\
\hline & MPZBEP & 2.132 & +0.007 & 1.3 \\
\hline \multicolumn{2}{|c|}{ Means Values } & $<2.3$ & -0.04 to +0.13 & $<11$ \\
\hline
\end{tabular}

${ }^{a}$ Compound contains Epz 4 as Tp ligand, and two times have been counted.

$b$ Two or more different conformations for a REpz 3 as Tp ligand. 
Table C4. Structural parameters and CSD refcodes for Tp compounds with structure of squareplanar coordination having the third pyrazolyl ring of Tp ligand over the metal (SP1).

\begin{tabular}{llll}
\hline Metal & Refcode & $d\left(\mathrm{M}^{-} \mathrm{L}_{a p}\right)$ & $d\left(\mathrm{M}-\mathrm{L}_{a p}\right)-d\left(\mathrm{M}-\mathrm{L}_{b s}\right) \quad \square\left(\mathrm{M}-\mathrm{E}-\mathrm{C}-\mathrm{N}_{a p}\right)$ \\
\hline
\end{tabular}

\section{Donor atoms}

\begin{tabular}{|c|c|c|c|c|}
\hline \multirow{2}{*}{\multicolumn{2}{|c|}{$\begin{array}{l}\operatorname{BIZTUZ~}^{a} \\
\text { BIZVAH }^{a}\end{array}$}} & 4.096 & +2.029 & 105.7 \\
\hline & & 3.694 & +1.595 & 95.0 \\
\hline & EKAFAX & 3.597 & +1.481 & 95.3 \\
\hline & " & 3.614 & +1.502 & 97.6 \\
\hline & EKAFIF & 3.484 & +1.334 & 107.0 \\
\hline & EKAFOL & 3.571 & +1.444 & 101.4 \\
\hline & GOFNEU & 3.641 & +1.559 & 93.4 \\
\hline & " & 3.644 & +1.550 & 92.5 \\
\hline & HIVHOJ & 3.799 & +1.707 & 101.3 \\
\hline & LOGVEI & 3.778 & +1.671 & 75.3 \\
\hline & MONWAN & 3.488 & +1.375 & 68.6 \\
\hline & NAMQOH & 3.633 & +1.526 & 97.5 \\
\hline & NANXOP & 3.901 & +1.808 & 102.3 \\
\hline & NOHBUH & 3.718 & +1.603 & 99.9 \\
\hline & $\mathrm{NOHCAO}^{b}$ & 3.390 & +1.264 & 78.7 \\
\hline & NORCEC & 3.782 & +1.669 & 99.4 \\
\hline & QETNEI & 3.925 & +1.800 & 103.4 \\
\hline & QETNIM & 3.854 & +1.737 & 105.0 \\
\hline & QETNOS & 3.747 & +1.646 & 101.7 \\
\hline & QETPAG & 3.700 & +1.595 & 75.2 \\
\hline & QETPEK & 3.713 & +1.613 & 94.6 \\
\hline & QOSREV & 3.535 & +1.439 & 92.9 \\
\hline & QOSROF & 3.513 & +1.417 & 87.6 \\
\hline & RUWZOY & 3.767 & +1.669 & 96.4 \\
\hline & RUWZUE & 3.709 & +1.610 & 93.8 \\
\hline & RUXBAN & 3.868 & +1.778 & 97.9 \\
\hline & TOKHAC01 & 3.538 & +1.423 & 89.9 \\
\hline & VIPVIZ & 3.918 & +1.834 & 101.0 \\
\hline & VIPVOF & 3.910 & +1.831 & 103.5 \\
\hline & VOQHOY & 3.661 & +1.565 & 91.4 \\
\hline & WAYNAL & 3.665 & +1.555 & 93.9 \\
\hline & WOGXEV & 3.677 & +1.577 & 94.9 \\
\hline & XAWQIV & 3.081 & +0.957 & 44.4 \\
\hline
\end{tabular}




\begin{tabular}{|c|c|c|c|c|}
\hline & XAWQOB & 3.420 & +1.323 & 57.2 \\
\hline & XAWQUH & 3.381 & +1.278 & 56.5 \\
\hline \multirow[t]{36}{*}{$\mathrm{Pd}$} & DIZHOJ & 3.384 & +1.387 & 84.9 \\
\hline & DIZHUP & 3.888 & +1.870 & 98.3 \\
\hline & EFUGER & 2.846 & +0.720 & 42.4 \\
\hline & EFUGIV & 3.336 & +1.248 & 87.1 \\
\hline & FUXKIS & 3.595 & +1.526 & 95.6 \\
\hline & " & 3.263 & +1.200 & 65.4 \\
\hline & GUVNOA $^{a}$ & 3.610 & +1.487 & 100.4 \\
\hline & $\mathrm{OGOZOZ}^{a}$ & $4.523^{c}$ & +2.445 & 121.0 \\
\hline & $"$ & $4.686^{c}$ & +2.591 & 126.5 \\
\hline & OGOZUF $^{a}$ & $4.751^{c}$ & +2.666 & 123.3 \\
\hline & $"$ & $4.816^{c}$ & +2.722 & 125.4 \\
\hline & OGUBAT $^{a}$ & $4.281^{c}$ & +2.224 & 116.2 \\
\hline & " & $4.358^{c}$ & +2.306 & 117.5 \\
\hline & PIHQEC & 3.808 & +1.771 & 101.2 \\
\hline & PIHQIG & 3.583 & +1.581 & 84.4 \\
\hline & PIHQOM & 3.486 & +1.447 & 88.1 \\
\hline & POCDOA & 4.597 & +2.519 & 137.4 \\
\hline & " & 4.357 & +2.281 & 125.7 \\
\hline & QIYWIE & 3.815 & +1.783 & 99.3 \\
\hline & QIYWOK & 3.524 & +1.521 & 86.5 \\
\hline & QIYWUQ & 3.497 & +1.489 & 88.7 \\
\hline & QIYXEB & 3.199 & +1.191 & 55.1 \\
\hline & QUQTUR & 3.388 & +1.383 & 74.1 \\
\hline & QUQVED & 3.483 & +1.468 & 80.6 \\
\hline & QUQVIH & 3.494 & +1.480 & 84.8 \\
\hline & QURDEM & 3.604 & +1.483 & 93.6 \\
\hline & " & 4.066 & +1.955 & 108.8 \\
\hline & RAVKOO01 & 3.066 & +0.988 & 59.4 \\
\hline & UHEVOS & 3.603 & +1.604 & 101.0 \\
\hline & " & 4.074 & +2.040 & 105.7 \\
\hline & UHEVUY $b$ & 3.949 & +1.944 & 105.7 \\
\hline & UHEWAF $b$ & 4.819 & +2.775 & 132.3 \\
\hline & YUMXUZ $^{a}$ & 3.816 & +1.701 & 94.9 \\
\hline & YUMYAG & 4.502 & +2.389 & 128.1 \\
\hline & YUMYEK $^{a}$ & 3.887 & +1.791 & 99.7 \\
\hline & YUMYIO & 4.506 & +2.414 & 115.1 \\
\hline $\mathrm{Ir}$ & NULNOX & 3.748 & +1.657 & 98.5 \\
\hline
\end{tabular}


$\mathrm{Pt}$

$$
\begin{aligned}
& \text { KEDYOH } \\
& \text { NOQSIV } \\
& \text { ODEDEG } \\
& \text { QAVQUZ } \\
& "
\end{aligned}
$$

QEHWUV

QEHXAC

QEHXEG

QEHXOQ

VODDAT

$\mathrm{Au}$

DAYCEL $^{a}$
DIRTAZ
FAGKUU $^{a}$

4.333

4.793

$3.605^{d}$

4.007

4.200

3.459

3.222

3.467

3.574

4.094

4.277

4.017

3.698

$$
+2.297
$$

$+2.686$

$+1.502$

$+1.944$

$+2.137$

$+1.376$

$+1.112$

$+1.374$

$+1.497$

$+2.088$

$+2.177$

$+1.919$

$+1.628$
120.6

128.8

92.2

95.6

106.6

91.6

71.5

86.2

91.9

110.8

85.3

90.0

97.3

\section{No donor atoms}

$\begin{array}{cc}\text { Rh } & \text { LEHFOT } \\ & \text { LEHFUZ } \\ & \text { ADOPOY } \\ & \text { GULRUA } \\ & " \\ & \text { GULSAH } \\ & \text { MOVLEO } \\ & \text { QAYFIF }\end{array}$

$$
\begin{aligned}
& 3.652,3.751 \\
& 3.488,3.863 \\
& 3.642,4.133 \\
& 3.292,3.706 \\
& 3.368,3.458 \\
& 3.623,3.915 \\
& 3.403,4.314 \\
& 3.666
\end{aligned}
$$$$
+1.563,1.661
$$$$
\text { 88.6, } 93.4
$$$$
+1.406,1.781
$$$$
79.2,101.0
$$$$
+1.529,2.020
$$$$
76.3,106.5
$$$$
+1.249,1.662
$$$$
80.9,104.9
$$$$
+1.326,1.416
$$$$
91.2,96.0
$$$$
+1.605,1.896
$$$$
82.0,98.5
$$$$
+1.310,2.221
$$$$
64.4,119.6
$$$$
+1.551
$$$$
91.6
$$

\section{Means Values}

2.8 to 4.8

+0.7 to +2.8

40 to 140

\footnotetext{
${ }^{a}$ Compound contains Epz 4 as Tp ligand, and has been counted two times.

$b$ Two or more different conformations for a $\mathrm{REpz}_{3}$.

${ }^{c}$ The third $N$-donor is coordinated to an other metallic center.

$d$ The nitrogen atom has been protonated.
} 
Table C5. Structural parameters and CSD refcodes for Tp compounds with structure of $\square^{2}$ squareplanar coordination for the metal atom (SP2).

\begin{tabular}{|c|c|c|c|}
\hline Metal & Refcode & $d\left(\mathrm{M}-\mathrm{L}_{a p}\right)$ & $d\left(\mathrm{M}-\mathrm{L}_{a p}\right)-d\left(\mathrm{M}-\mathrm{L}_{b s}\right)$ \\
\hline \multirow[t]{7}{*}{$\mathrm{Rh}$} & BIZTUZ $^{a}$ & 5.865 & +3.798 \\
\hline & $\mathrm{BIZVAH}^{a}$ & 5.572 & +3.977 \\
\hline & BIZVEL $^{a}$ & 5.474 & +3.368 \\
\hline & " & 5.575 & +3.483 \\
\hline & NANXUV & 5.395 & +3.301 \\
\hline & QOSRAR & 5.300 & +3.236 \\
\hline & TOXGOC & 5.384 & +3.290 \\
\hline \multirow[t]{14}{*}{$\mathrm{Pd}$} & GUVNOA $^{a}$ & 5.426 & +3.304 \\
\hline & HIMZUY & 5.609 & +3.495 \\
\hline & HINBAH & 5.522 & +3.439 \\
\hline & MEXRUC & $4.542^{b}$ & +2.508 \\
\hline & MEXSAJ & $4.310^{b}$ & +2.249 \\
\hline & " & $4.351^{b}$ & +2.281 \\
\hline & $\mathrm{OGOZOZ}^{a}$ & $5.856^{c}$ & +3.779 \\
\hline & " & $5.871^{c}$ & +3.776 \\
\hline & $\mathrm{OGOZUF}^{a}$ & $5.924^{c}$ & +3.840 \\
\hline & " & $5.976^{c}$ & +3.882 \\
\hline & OGUBAT $^{a}$ & $5.686^{c}$ & +3.629 \\
\hline & " & $5.712^{c}$ & +3.661 \\
\hline & YUMXUZ $^{a}$ & 5.555 & +3.439 \\
\hline & YUMYEK $^{a}$ & 5.544 & +3.448 \\
\hline Ir & OCUXAL & 5.504 & +3.423 \\
\hline $\mathrm{Pt}$ & MPZBPT10 $d$ & $5.516,5.834$ & $+3.472,3.791$ \\
\hline \multirow[t]{2}{*}{$\mathrm{Au}$} & $\operatorname{DAYCEL}^{a}$ & 5.506 & +3.407 \\
\hline & FAGKUU $^{a}$ & 5.511 & +3.441 \\
\hline \multicolumn{2}{|c|}{ Means Values } & $>4.3$ & $>2.2$ \\
\hline
\end{tabular}

${ }^{a}$ Compound contains $\mathrm{Epz}_{4}$ as Tp ligand, and it has been counted two times.

${ }^{b}$ The third $N$-donor is coordinated with a weak $\mathrm{Pd} \cdots \mathrm{Pd}$ interaction.

${ }^{c}$ The third $N$-donor is coordinated to an other metallic center.

$d$ Disorder between the positions 2 and 5 of the third pyrazolyl ring. 